\title{
Effect of electric field on exfoliation of nanoplates
}

\author{
Wei Lu ${ }^{\mathrm{a})}$ \\ Department of Mechanical Engineering, University of Michigan, Ann Arbor, Michigan 48109 \\ Hilmar Koerner and Richard Vaia \\ Air Force Research Laboratory, Materials and Manufacturing Directorarte, AFRL/MLBP, Bldg 654, \\ 2941 Hobson Way, Wright-Patterson AFB, Ohio 45433
}

(Received 14 September 2006; accepted 16 October 2006; published online 30 November 2006)

\begin{abstract}
Nanocomposite performance relies on reproducible dispersion and arrangement of nanoparticles, such that the dominant morphology across macroscopic dimensions is nanoscopic. The impact of electric fields on the interaction of nanoplates is discussed for improving dispersion. For ideal dielectrics, an electric field may assist (or retard) exfoliation depending on the angle between a collection of plates and the field. A critical electric field strength for exfoliation is predicted. Structural refinement occurs by cleavage through the center of the stack. For lossy dielectrics, frequency can be tuned to cause exfoliation in all plate orientations. (C) 2006 American Institute of Physics. [DOI: 10.1063/1.2398913]
\end{abstract}

Polymeric materials with multiphase morphologies on the nanoscale $(1-100 \mathrm{~nm})$ and mesoscale $(100-500 \mathrm{~nm})$ offer exciting opportunities for high-end, multifunctional applications, as well as enabling never-before-realized materials with unique combinations of electrical, optical, and structural properties. To facilitate dispersion, chemical approaches, including surfactant or macromolecular stabilization are usually employed to modify the surface of nanoparticles. However, the approach depends on the material system and usually involves trial and error to identify the best practice. Much less quantitative information is available on the coupling between the surface modification and external processing factors, including shear, ${ }^{1}$ electric, ${ }^{2}$ or magnetic ${ }^{3}$ fields. This letter discusses a physical mechanism based on the application of an external electric field to effect the agglomeration of nanoplates.

Due to the strength and long range of electrostatic interactions, electric fields have been used to control structures on length scales which are difficult to manipulate in other manners. For instance, it has been used to move or separate microparticles, ${ }_{8}^{2,4}$ trap nanospheres, ${ }^{6}$ align nanowires, ${ }^{7}$ pattern molecules, ${ }^{8}$ and control the morphology of polymer melts. ${ }^{9,10}$ While dielectrophoresis has been used to separate microparticles, this effect relies on field gradient and different dielectric properties of the particles. In contrast, if a composite particle is comprised of low-dimensional regions loosely bonded together, such as the interlayer regions within a layer stack, an application of a uniform field can generate internal forces within the particle. The analysis shows that for ideal dielectrics, an electric field may assist (repulsion) or retard (attraction) exfoliation depending on the angle between the plate and the field. Exfoliation is promoted when the field is parallel to the plate surface, providing a possible avenue to address the dispersion challenge in nanocomposites. The study of lossy dielectrics suggests that tuning the frequency could lead to exfoliation in all plate orientations.

The effect of electric field on nanoparticle interaction can be understood in terms of energetics. Without losing generality, consider the application of a uniform electric field $\mathbf{E}_{0}$ to two plates (perfect dielectrics) in vacuum, separated by $r$

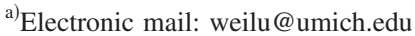

(Fig. 1). When the plates are far apart, the dipole interaction energy scales as $E_{0}^{2} / r^{3}\left(-E_{0}^{2} / r^{3}\right)$ in the parallel (perpendicular) configuration. Energy reduction drives an increase (decrease) in $r$ or repulsion (attraction) between the plates. Similar trends hold when two plates are very close, though the $1 / r^{3}$ dependence does not apply. Two plates at an arbitrary orientation [Fig. 1(c)] can be understood by the superposition of these attractive and repulsive configurations. In addition to interparticle interaction, the induced dipole will interact with $\mathbf{E}_{0}$ to cause the plate orient its longer axis along $\mathbf{E}_{0}$. In the absence of field gradients, this will eventually lead to an all-parallel configuration. Thus at sufficient times, a repulsive force between plates should drive exfoliation and homogenization of the collection of aligned plates.

Quantitatively, what are the limits to homogenization driven by these induced dipoles? What is the role of the dielectric property and particle geometry as well as applied field strength and frequency? What is the internal energy and failure criterion of an agglomerate? To develop an understanding of this process, initially the interactions between two ideal plates in an ideal dielectric and the field magnitude necessary to cause particle cleavage are discussed. This is then extended to lossy dielectrics.

Consider a circular plate of diameter $d$ and thickness $t$ (Fig. 1) that is allowed to separate into two thinner plates, each with thickness $t / 2$. We choose the energy reference state to be the media under an applied field $\mathbf{E}_{0}$. When part of the media is replaced by a plate, the energy change defines the free energy $G_{e}$. For linear dielectrics, the free energy is

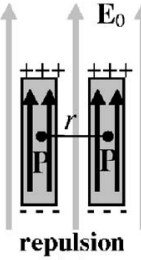

(a)

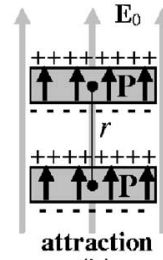

(b)

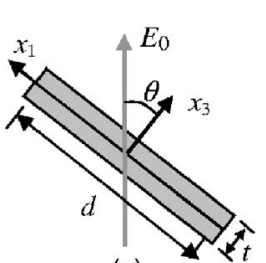

(c)
FIG. 1. Conceptual extrema for circular plates of diameter $d$ and thickness $t$ (a) parallel (attraction) and (b) perpendicular (repulsion) to the applied field $\mathbf{E}_{0}$. (c) A coordinate is attached so that the plate lies in the $x_{1}-x_{2}$ plane with $x_{3}$ normal to the surface. Plate orientation is described by an angle $\theta$. 


$$
G_{e}=-\frac{1}{2} \int_{V_{p}}\left(\varepsilon_{p}-\varepsilon_{m}\right) \mathbf{E} \cdot \mathbf{E}_{0} d V
$$

where $\mathbf{E}$ is the electric field in the plate, $V_{p}$ the plate volume, $\varepsilon_{p}$ the permittivity of the plate, and $\varepsilon_{m}$ the permittivity of the fluidic media. Note that $\mathbf{E}$ is usually different from $\mathbf{E}_{0}$ in both magnitude and direction. Equation (1) applies to the situation that $\varepsilon_{p}(\mathbf{x})$ is an arbitrary function of position $\mathbf{x}$, where the integration extends over the entire domain. Thus the expression can be used to calculate the energy of multiple plates, where $V_{p}$ stands for the total plate volume since $\varepsilon_{p}$ $-\varepsilon_{m}$ vanishes in the media. For a single plate shown in Fig. $1(\mathrm{c})$, the free energy is

$$
\begin{aligned}
G_{e}= & -\frac{1}{2} \varepsilon_{m} V_{p} E_{0}^{2}\left[g_{1}\left(t / d, \varepsilon_{p} / \varepsilon_{m}\right) \sin ^{2} \theta\right. \\
& \left.+g_{3}\left(t / d, \varepsilon_{p} / \varepsilon_{m}\right) \cos ^{2} \theta\right],
\end{aligned}
$$

where $g_{1}$ and $g_{3}$ are two dimensionless functions relevant to the aspect $t / d$ and permittivity $\varepsilon_{p} / \varepsilon_{m}$ ratio. The dependence on the plate orientation is obtained by projecting the applied field parallel and perpendicular to the plate surface. Thus the orientation dependence in Eq. (2) is the same for arbitrary plate geometry and dielectric property.

The application of Eqs. (1) and (2) to two plates gives the distance dependent interactive energy. Analytic expressions are available at the two limits: agglomerated plates and fully exfoliated plates, and our numerical analysis indicates that the interaction decays very fast within a few $t$. The electric potential obeys the Laplace equation, which can be solved analytically in ellipsoidal coordinates. Under a uniform field $\mathbf{E}_{0}$, the electric field in an ellipsoid $\mathbf{E}$ is uniform, though the field in the media is complicated. Substituting $\mathbf{E}$ into Eqs. (1) and (2) gives

$$
\begin{aligned}
& g_{1}\left(t / d, \varepsilon_{p} / \varepsilon_{m}\right)=\frac{\varepsilon_{p} / \varepsilon_{m}-1}{1+S_{1}\left(\varepsilon_{p} / \varepsilon_{m}-1\right)}, \\
& S_{1}=\frac{1}{2} \int_{0}^{\infty} \frac{d \xi}{(1+\xi)^{2} \sqrt{1+\xi(d / t)^{2}}}, \\
& g_{3}\left(t / d, \varepsilon_{p} / \varepsilon_{m}\right)=\frac{\varepsilon_{p} / \varepsilon_{m}-1}{1+S_{3}\left(\varepsilon_{p} / \varepsilon_{m}-1\right)}, \\
& S_{3}=\frac{1}{2} \int_{0}^{\infty} \frac{d \xi}{(1+\xi)\left(\xi+(t / d)^{2}\right) \sqrt{1+\xi(d / t)^{2}}},
\end{aligned}
$$

where $S_{1}$ and $S_{3}$ are shape factors relevant to the aspect ratio $t / d$ of the ellipsoid which approximates the plate.

The energy change upon exfoliation $\Delta G_{e}$ is the difference between the energy of a single plate and that of two thinner isolated plates, each having half of the original thickness, namely,

$\Delta G_{e}=-\frac{1}{2} \varepsilon_{m} V_{p} E_{0}^{2}\left[R_{1}\left(t / d, \varepsilon_{p} / \varepsilon_{m}\right) \sin ^{2} \theta+R_{3}\left(t / d, \varepsilon_{p} / \varepsilon_{m}\right) \cos ^{2} \theta\right]$,

where $\quad R_{1}=g_{1}\left(t /(2 d), \varepsilon_{p} / \varepsilon_{m}\right)-g_{1}\left(t / d, \varepsilon_{p} / \varepsilon_{m}\right) \quad$ and $\quad R_{3}$ $=g_{3}\left(t /(2 d), \varepsilon_{p} / \varepsilon_{m}\right)-g_{3}\left(t / d, \varepsilon_{p} / \varepsilon_{m}\right)$ are two dimensionless functions relevant to the geometry and dielectric property.

The dependence of $R_{1}$ and $R_{3}$ curves on the aspect and permittivity ratios are shown in Fig. 2. The $R_{1}$ term essentially describes the energy change of the parallel configura-

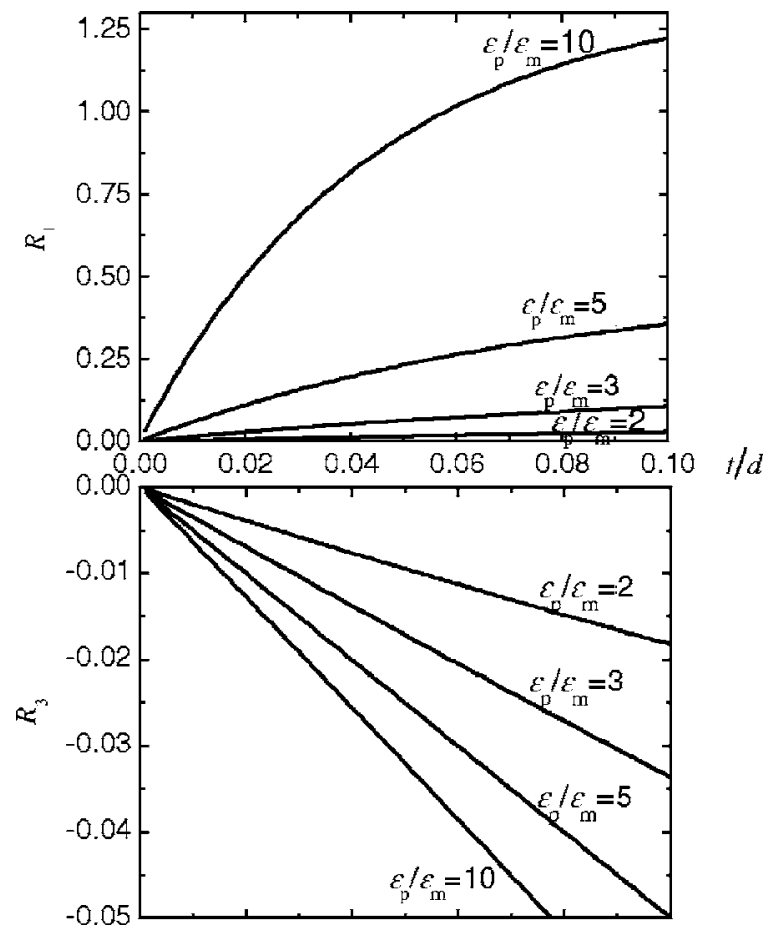

FIG. 2. For an ideal dielectric, the impact of aspect ratio on the dimensionless parameters $R_{1}$ and $R_{3}$ (Eq. (5)). $R_{1}$ and $R_{3}$ are proportional to the energy change in the parallel and perpendicular configurations, respectively. Positive values suggest exfoliation reduces free energy, and is preferred.

tion, i.e., $\theta=\pi / 2$. Note that $R_{1}$ is positive, which suggests that exfoliation reduces the free energy, and thus is preferred. The perpendicular configuration corresponds to $\theta=0$ and only $R_{3}$ acts. In this case, the negative $R_{3}$ term promotes agglomeration. These features are consistent with the prior conceptual discussion. The magnitudes of $R_{1}$ and $R_{3}$ increase with aspect ratio. This trend is anticipated since larger $t / d$ means more material distributed in the thickness direction contributing to an edge effect. Additionally, the magnitudes increase with $\varepsilon_{p} / \varepsilon_{m}\left(\varepsilon_{p} / \varepsilon_{m}>1\right)$. Finally, for any given aspect ratio and $\varepsilon_{p} / \varepsilon_{m}>1$, the magnitude of $R_{1}$ is much larger than that of $R_{3}$, suggesting a dominating repulsion. For the situation of $\varepsilon_{p} / \varepsilon_{m}<1$ smaller $\varepsilon_{p} / \varepsilon_{m}$ also leads to increased magnitudes of $R_{1}$ and $R_{3}$; however, the magnitude of $R_{1}$ is much smaller than $R_{3}$. For instance, $\varepsilon_{p} / \varepsilon_{m}=0.1$ and $t / d$ $=0.1$ lead to $R_{1}=0.029$ and $R_{3}=-1.4$, suggesting a dominat ing attraction. These results indicate that electric fields can be used to help exfoliation, especially when the plates have permittivity larger than that of the media. Equation (5) shows a quadratic dependence on the field strength.

Although the impact of arbitrary geometry requires numerical calculation, an analytic result can be obtained when the in-plane shape is approximated by an ellipse, leading to lathe-like particles, such as $\mathrm{V}_{2} \mathrm{O}_{5}$. Relative to the field direction, vary the plate shape (width $b)$ from $\operatorname{sim}(b=d / 2)$ to wide $(b=2 d)$, where a circulate plate has $b / d=1$. Generally, the shape irregularity is insignificant for thin plates with permittivity close to that of the media. The effect increases for thicker plates and larger $\varepsilon_{p} / \varepsilon_{m}$. Nevertheless, for a wide range of parameters considered $\left(\varepsilon_{p} / \varepsilon_{m}, t / d\right)$, the difference of $R_{1}$ from that of a circular plate is less than $15 \%$.

For nonideal dielectrics, conduction and dielectric relaxations lead to a frequency-dependent complex dielectric constant. In this case, the dielectric properties in Eqs. (3) and (4) 


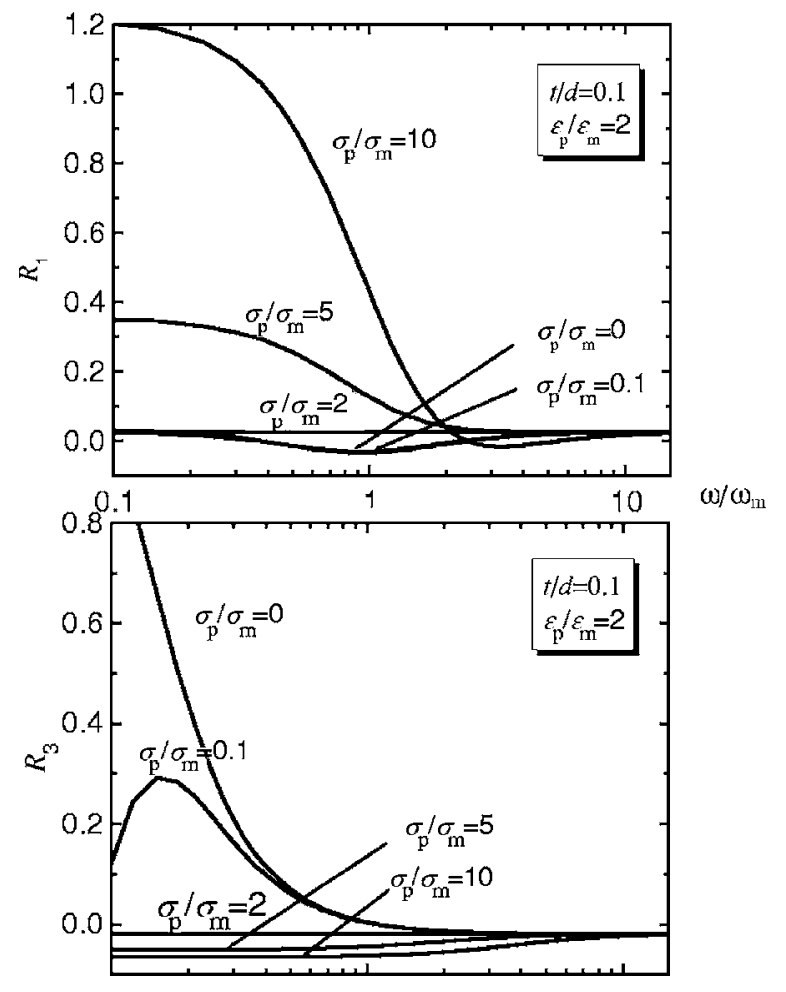

FIG. 3. For a lossy dielectric, the impact of relative conductivity on the magnitude and sign of $R_{1}$ and $R_{3}$ as a function of normalized frequency $\omega / \omega_{m}$

are replaced with $\varepsilon_{p}^{*}(\omega)=\varepsilon_{p}-i \sigma_{p} / \omega$ and $\varepsilon_{m}^{*}(\omega)=\varepsilon_{m}-i \sigma_{m} / \omega$, where $\omega$ is the electric field frequency and $\sigma_{p}$, and $\sigma_{m}$ are the electric conductivities of the plate and media. The characteristic frequency is $\omega_{m}=\sigma_{m} / \varepsilon_{m}$. Figure 3 shows $R_{1}$ and $R_{3}$ as a function of normalized frequency for representative aspect ratio, $t / d=0.1$, and permittivity ratio, $\varepsilon_{p} / \varepsilon_{m}=2$. The behavior becomes frequency independent when $\varepsilon_{p} / \varepsilon_{m}=\sigma_{p} / \sigma_{m}$. At high frequency when charge accumulation cannot respond quickly enough, all the curves converge to the case of an ideal dielectric. Figure 3 suggests that the impact of finite conduction is important when the frequency is comparable to $\omega_{m}$. For instance, $R_{1}$ is 0.029 for ideal dielectrics of $\varepsilon_{p} / \varepsilon_{m}$ $=2$, but increases to 1.22 at $\sigma_{p} / \sigma_{m}=10$ and frequency of $0.1 \omega_{m}$. This magnitude is comparable to the situation of ideal dielectrics with $\varepsilon_{p} / \varepsilon_{m}=10$. Also, important to note is that when $\varepsilon_{p}>\varepsilon_{m}$ and $\sigma_{p}<\sigma_{m}$, a frequency window occurs where the sign of $R_{1}$ and $R_{3}$ reverses. This behavior suggests the possibility of frequency tuning to change the interaction of two plates and promote exfoliation for any plate orientation.

The prior analysis implies that an electric field can be applied to prevent the agglomeration of dispersed nanoplates during mixing. If the plates are already agglomerated, will the induced polarization produce sufficient internal energy to cause exfoliation? Exfoliation of a low-dimensional crystal involves cleavage along a plane of weak secondary bonding, a process that is conceptually analogous to crack extension. Separation occurs when the energy release rate overcomes the resistance due to the creation of new surfaces. Phenom- enologically, the interfacial energy is defined as $2 \Gamma A$, where $2 A$ is the interfacial area and $\Gamma$ is the interfacial energy. Separation of plates requires $\Delta G_{e}>2 \Gamma A$. Repulsion maximizes at $\theta=\pi / 2$ and leads to a critical field,

$$
E_{C}^{2}=\frac{4 \Gamma}{\varepsilon_{m} t R_{1}} .
$$

For larger fields $\left(E>E_{C}\right)$, exfoliation is advantageous for $\theta$ $<\theta_{C}$ defined by $\sin ^{2} \theta_{c}=\left(4 \Gamma /\left(\varepsilon_{m} E^{2} t\right)-R_{3}\right) /\left(R_{1}-R_{3}\right)$. For brittle fracture in vacuum, $\Gamma$ is half the work of cohesion and equivalent to the specific surface energy of the solid, $\gamma_{\text {solid }}$. For organically modified montmorillonite, $\gamma_{\text {solid }}$ $\sim 40-50 \mathrm{~mJ} / \mathrm{m}^{2}$ for the surfactant covered basal plane. Therefore nanoplates with thickness of $10 \mathrm{~nm}$ and $t / d$ $\sim 0.1-0.01$ require a field of 350 to $800 \mathrm{~V} / \mu \mathrm{m}$. Calculations show that the energy relaxes more when a body breaks into two equal pieces. Thus an applied field will cause a multiplate agglomeration to break in the middle rather than peeling off layers from the outside.

The estimated field strength in vacuum is comparable to the dielectric breakdown strength of organics and polymers. However, in liquid medium, the total interfacial energy will be less than that in vacuum in accordance with the Young relation describing surface wetting. The maximum change occurs when spreading is favored (contact angle $=0$ ) and thus $\Gamma \sim \gamma_{\text {solid }} \gamma_{\text {liquid. }}$ Equation (6) then provides the critical field that induced polarization may overcome surface energy differences between the matrix and nanoplate to enable exfoliation. Putting this into perspective with the experimental data of layered silicate nanocomposites such as nylon 6 and $6 / 6$ as well as n-methyl-2-pyrrolidone known to spontaneously exfoliate many organically modified montorillonites, these systems reveal values of $\gamma_{\text {solid }}-\gamma_{\text {liquid }} \sim 0-5 \mathrm{~mJ} / \mathrm{m}^{2} . \gamma_{\text {solid }}$ $-\gamma_{\text {liquid }}$ exhibits a wide range in actual nanocomposite systems and establishes an $E_{\mathrm{C}}$ for the experimentalist to enable exfoliation.

In summary, free-energy based considerations indicate that nanoplate interactions can be modified under a sufficient applied electric field $(>1-10 \mathrm{~V} / \mu \mathrm{m})$, leading to situations in which induced polarization produces an additional repulsive potential. The magnitude of this additional potential, for both ideal and lossy dielectrics, is sufficient to provide experimental control to assist (or retard) exfoliation.

${ }^{1}$ A. B. D. Brown and A. R. Rennie, Phys. Rev. E 62, 851 (2000).

${ }^{2}$ N. G. Green, A. Ramos, and H. Morgan, J. Phys. D 33, 632 (2000).

${ }^{3}$ H. Koerner, E. Hampton, D. Dean, Z. Turgut, L. Drummy, P. Mirau, and R. Vaia, Chem. Mater. 17, 1990 (2005).

${ }^{4}$ A. Docoslis and P. Alexandridis, Electrophoresis 23, 2174 (2002).

${ }^{5}$ J. Rousselet, L. Salome, A. Ajdari, and J. Prost, Nature (London) 370, 446 (1994).

${ }^{6}$ T. Schnelle, T. Müller, G. Gradl, S. G. Shirley, and G. Fuhr, Electrophoresis 21, 66 (2000).

${ }^{7}$ P. A. Smith, C. D. Nordquist, T. N. Jackson, T. S. Mayer, B. R. Martin, J. Mbindyo, and T. E. Mallouk, Appl. Phys. Lett. 77, 1399 (2000).

${ }^{8}$ W. Lu and D. Salac, Phys. Rev. Lett. 94, 146103 (2005).

${ }^{9}$ E. Schäffer, T. Thurn-Albrecht, T. P. Russell, and U. Steiner, Nature (London) 403, 874 (2000).

${ }^{10}$ D. Kim and W. Lu, Phys. Rev. B 73, 035206 (2006). 